Chronic Obstructive Pulmonary Diseases: Journal of the COPD Foundation

\author{
Original Research
}

\title{
Long-Term Maintenance Bronchodilation With Indacaterol/ Glycopyrrolate Versus Indacaterol in Moderate-to-Severe COPD Patients: The FLIGHT 3 Study
}

Gary T. Ferguson, $\mathrm{MD}^{1}$ Angel Fowler Taylor, BSc, Pharm ${ }^{2}$ Chau Thach, $\mathrm{PhD}^{2}$ Qian Wang, $\mathrm{PhD}^{3}$ Agnes A. Schubert-Tennigkeit, $\mathrm{MD}^{4}$ Francesco Patalano, $\mathrm{MD}^{4}$ and Donald Banerji, $\mathrm{MD}^{2}$

\section{Abstract}

Background: The objective of the FLIGHT3 study was to evaluate the long-term safety and efficacy of indacaterol/ glycopyrrolate* (IND/GLY) versus an active comparator, IND, in patients with moderate-to-severe chronic obstructive pulmonary disease (COPD) over 52 weeks.

Method: FLIGHT3 was a multicenter, randomized, double-blind, parallel-group, 52-week study. Patients were

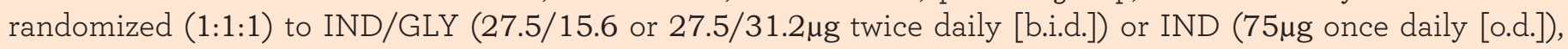
delivered via the Neohaler ${ }^{\circledR}$ device. The primary objective was to evaluate the long-term safety and tolerability of IND/GLY versus IND in terms of adverse event (AE)-reporting rates in patients with moderate-to-severe COPD over 52 weeks. The secondary objective was to evaluate the long-term efficacy of IND/GLY versus IND in terms of pre-dose trough forced expiratory volume in 1 second $\left(F^{2} V_{1}\right)$ and post-dose 1 -h FEV 1 over 52 weeks.

Results: A total of $\mathbf{8 5 . 2 \%}$ patients completed the study treatment. The overall incidence of AEs (and SAEs) was similar between treatments. Major adverse cardiovascular events (MACE) and/or cardiovascular (CV) events were comparable between treatment groups. The rate of discontinuation of the study treatment due to AEs was lower for IND/GLY than IND. Improvements in pre-dose trough $\mathrm{FEV}_{1}$ and post-dose 1 - $\mathrm{h}$ FEV 1 were consistently superior with IND/GLY than with IND over 52 weeks, demonstrating long-term maintenance of lung function.

Conclusions: IND/GLY demonstrated a favorable long-term safety and tolerability profile and provided effective bronchodilation, with maintenance of lung function over 52 weeks in patients with moderate-to-severe COPD. These data support the safety and efficacy of IND/GLY as a treatment option for COPD.

Trial registration: ClinTrials.gov identifier NCT01682863

*Glycopyrrolate $15.6 \mu \mathrm{g}$ (excluding the bromide salt) is equivalent to $12.5 \mu \mathrm{g}$ glycopyrronium

\begin{abstract}
Abbreviations: indacaterol, IND; glycopyrrolate, GLY; chronic obstructive pulmonary disease, COPD; twice daily, B.I.D; once daily, O.D.; adverse event, $\mathbf{A E}$; forced expiratory volume in 1 second, $\mathbf{F E V}_{\mathbf{1}}$; major adverse cardiovascular events, $\mathbf{M A C E}$; cardiovascular, $\mathbf{C V}$; inhaled corticosteroids, ICS; long-acting beta2-agonists, LABA; long-acting muscarinic antagonist, LAMA; fixed dose combination, FDC; Food and Drug Administration, FDA; Global initiative for chronic Obstructive Lung Disease, GOLD; forced vital capacity, FVC; modified Medical Research Council, mMRC; Interactive Response Technology, IRT; electrocardiogram, ECG; serious adverse event, SAE; cerebrovascular, CCV; Medical Dictionary for Regulatory Activities, MedDRA; mixed-model repeated-measures, MMRM; full analysis set, FAS; linear mixed model, LMM; COPD assessment test, CAT; hazard ratio, HR

Funding Support: Funding for this project was provided by Novartis Pharmaceuticals, Corp.

Date of Acceptance: May 18, 2016

Citation: Ferguson GT, Taylor AF, Thach C, et al. Long-term maintenance bronchodilation with indacaterol/glycopyrrolate versus indacterol in modertate-to-severe COPD patients: The Flight3 study. Chronic Obstr Pulm Dis (Miami). 2016;3(4):716-728. doi: http://dx.doi. org/10.15326/jcopdf.3.4.2016.0131
\end{abstract}

This article has an online supplement. 
1 Pulmonary Research Institute of Southeast Michigan-Farmington
Hills 2 Novartis Pharmaceuticals Corporation, East Hanover, New Jersey

3 Beijing Novartis Pharma Co. Ltd., Shanghai, China

4 Novartis Pharma AG, Basel, Switzerland

\section{Address correspondence to:}

Gary T. Ferguson, MD

Pulmonary Research Institute of Southeast Michigan

29255 West 10 Mile Road, Suite A

Farmington Hills, Michigan 48336

E-mail: garytferguson@msn.com

Phone: (248) 350-2722

\section{Keywords:}

chronic obstructive pulmonary disease; COPD; long-acting beta2-agonists; long-acting muscarinic antagonist; clinical trials

\section{Introduction}

Chronic obstructive pulmonary disease (COPD) is a progressive debilitating respiratory condition characterized by respiratory symptoms with persistent and progressive airflow limitation. ${ }^{1}$ It is a major cause of poor health and death worldwide and contributes significantly to health care costs and comorbidity. ${ }^{2-4}$ COPD remains a significant public health challenge and is one of the leading causes of morbidity and mortality in the United States, affecting an estimated 27 million Americans. ${ }^{5}$ It is currently the third leading cause of death in the United States and claimed 134,676 lives in $2010 .^{6}$ It results in 1.5 million visits to emergency departments, over 15 million physician visits every year and 739,000 hospitalizations. 7,8

COPD is a treatable disease. However, due to the heterogeneity of the disease and its response to current therapies, new therapeutic options are needed. Current COPD treatment guidelines recommend the use of one or more bronchodilators, depending on the severity of patients' airflow limitations, history of exacerbations, and symptoms. ${ }^{1}$ Inhaled bronchodilator therapies, such as selective beta2-adrenergic receptor agonists and muscarinic receptor antagonists, are widely used treatment options for COPD, as are inhaled corticosteroids (ICS) in combination with a long-acting beta2-agonists (LABA). ${ }^{1}$ Studies have demonstrated that complementary mechanisms of action of a LABA and a long-acting muscarinic antagonist (LAMA) significantly improve bronchodilation in COPD patients compared with the respective monotherapy components. ${ }^{9,10}$ Combining bronchodilators, each with a different mechanism of action to influence airflow limitation, may maximize the bronchodilator response and impact the inter- and intra-patient variability in bronchometer tone associated with COPD. ${ }^{11,12}$ In addition, several studies have demonstrated that LABA/LAMA combinations have a better impact on lung function, inspiratory capacity, and quality of life compared with the respective monotherapies. ${ }^{13-15}$ Indacaterol maleate/glycopyrrolate (IND/GLY; QVA149) is a fixed-dose combination (FDC) of a LABA (indacaterol [IND]; QAB149) and a LAMA (glycopyrrolate [GLY]; NVA237) intended to be used for the long-term maintenance treatment of airflow obstruction in patients with COPD including chronic bronchitis and/or emphysema. The safety and efficacy of both IND and GLY have been demonstrated, with each monocomponent having a safety profile similar to that of a placebo. ${ }^{16-18}$

FDC therapy may also offer a potential advantage over monotherapy by increasing treatment adherence through the use of a single inhaler. Patients using multiple inhalers have demonstrated higher risk of exacerbations along with significantly higher health care costs and utilization. ${ }^{20}$ IND/GLY provides the opportunity for patients with COPD to receive dual bronchodilator therapy using 1 device, which results in rapid bronchodilation and a sustained efficacy for 12 hours. $^{21}$ In December 2013, the Food and Drug Administration (FDA) approved the first dual longacting bronchodilator (Anoro ${ }^{\circledR}$ Ellipta ${ }^{\circledR}$ ) for the longterm maintenance treatment of airflow obstruction in patients with COPD including chronic bronchitis and emphysema. Stiolto ${ }^{\mathrm{TM}}$ Respimat $^{\oplus}$, a once-daily (o.d.) FDC of tiotropium and olodaterol, was approved in June 2015 by the FDA for treatment of COPD. In addition, the FDA has recently approved the dual combination (IND/ GLY) Utibron Neohaler ${ }^{\mathrm{TM}}$ for the long-term maintenance treatment of airflow obstruction in patients with COPD.

The purpose of the FLIGHT3 study was to demonstrate the long-term safety and tolerability of twice-daily (b.i.d.) IND/GLY compared with an active comparator, IND, administered o.d. in patients with moderate-tosevere COPD.

\section{Methods}

\section{Study Design}

FLIGHT3 was a multicenter, randomized, doubleblind, parallel-group study that compared safety and 
tolerability over a 52-week period (ClinTrials.gov identifier: NCT01682863). The first patients were enrolled on October 26, 2012, and the study was completed on June 30, 2014. Patients were screened across 88 centers in 6 countries: Bulgaria (5), Finland (4), Hungary (10), Romania (10), Spain (8), and the United States (51). The study complied with International Conference on Harmonisation (ICH) Harmonised Tripartite Guidelines for Good Clinical Practice, applicable local regulations, and ethical principles laid down in the Declaration of Helsinki.

\section{Patients}

The study recruited male and female patients aged $\geq 40$ years who had stable COPD according to the 2011 Global initiative for chronic Obstructive Lung Disease (GOLD) criteria. ${ }^{1}$ Patients were included if they had moderate-to-severe airflow limitation, as indicated by post-bronchodilator forced expiratory volume in 1 second $\left(\mathrm{FEV}_{1}\right) \geq 30 \%$ and $<80 \%$ of the predicted normal and a post-bronchodilator $\mathrm{FEV}_{1}$ /forced vital capacity (FVC) ratio $<0.70$ at run in. The patients were either current or ex-smokers, with a smoking history of at least 10 pack years, and were symptomatic, as defined by a modified Medical Research Council (mMRC) dyspnea scale, Grade $\geq 2$.

Patients with any history of asthma or concomitant pulmonary disease or with a significant disease other than COPD that could significantly confound the trial results or preclude trial completion (including cardiovascular [CV], neurological, endocrine, immunological, psychiatric, gastrointestinal, hepatic, or hematological abnormalities) were excluded. Patients were also excluded if they had a COPD exacerbation that required treatment with antibiotics and/or systemic corticosteroids and/or hospitalization in the 6 weeks prior to Visit 1. Further information on exclusion criteria is available in the online supplementary data (eTable 1). All patients provided written informed consent, and all protocols were approved by an ethics committee.

\section{Study Treatments and Randomization}

This study comprised a 7-day screening period and a 14-day run-in period, followed by randomization 1:1:1

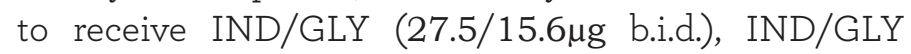

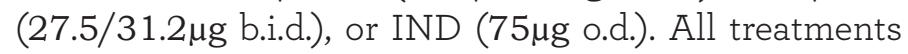
were delivered via the Neohaler ${ }^{\mathrm{TM}}$ device. The randomized, multicenter, double-blind design of this study was selected to provide rigor and reduce bias in addressing the long-term safety of the FDCs IND/GLY $27.5 / 15.6$ and $27.5 / 31.2 \mu \mathrm{g}$ b.i.d. versus IND $75 \mu \mathrm{g}$ o.d. A placebo group was not included because of ethical concerns based on the severity of patients studied and the study duration. The patients were treated for 52 weeks in order to establish the long-term safety of IND/ GLY. The inclusion of IND $75 \mu$ g o.d. as a comparator allowed for the comparison of the safety profile of IND/ GLY with that of IND, an approved treatment for COPD in the United States. ${ }^{22}$ A higher dose of the FDC IND/ GLY 27.5/31.2 $\mu$ g b.i.d, with the LAMA component being

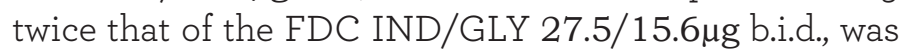
included as a treatment group to obtain additional safety data. The dose and dosing regimen of IND are based on the approved dose in the United States and supported by a previous study (NCT01959412). The dose and dosing regimen of GLY are based on an initial doseranging study (NCT00501852), ${ }^{22}$ which investigated o.d. doses of GLY, and an additional dose-ranging study (NCT01119950), ${ }^{23}$ which included multiple o.d. and b.i.d. doses.

All eligible patients were randomized via Interactive Response Technology (IRT) to one of the treatment groups. Treatment randomization was stratified based on the smoking status, ICS use, and severity of airflow limitation. Patients were instructed to take 1 capsule each morning (between 07:00 and 11:00 h) and 1 in the evening (between 19:00 and 23:00 h). Each patient was provided with a salbutamol/albuterol inhaler, which was permitted for use as rescue medication throughout the study. Nebulized salbutamol/albuterol was not permitted. Patients used an electronic diary (e-diary) to capture the use of rescue medication.

\section{Outcomes and Assessments}

The primary objective was to evaluate the safety and

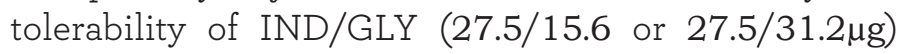
versus IND $(75 \mu \mathrm{g})$ in terms of adverse event (AE)reporting rates in patients with moderate-to-severe COPD over 52 weeks. Secondary objectives were to evaluate the bronchodilator effect of IND/GLY $(27.5 / 15.6$ or $27.5 / 31.2 \mu g)$ versus IND $(75 \mu \mathrm{g})$ on the mean $\mathrm{FEV}_{1}$ at pre-dose 15 and 45 minutes (predose trough $\mathrm{FEV}_{1}$ ) at Week 52 and on $\mathrm{FEV}_{1}$ and FVC at all post-baseline time points (including post-dose $1 \mathrm{~h})$. Other secondary objectives were to evaluate the safety and tolerability of IND/GLY (27.5/15.6 or $27.5 / 31.2 \mu \mathrm{g})$ versus IND $(75 \mu \mathrm{g})$ in terms of vital signs, electrocardiogram (ECG), laboratory evaluations, and 
treatment discontinuation and the effect of IND/GLY $(27.5 / 15.6$ or $27.5 / 31.2 \mu \mathrm{g})$ versus IND $(75 \mu \mathrm{g})$ on time to first moderate or severe exacerbation, COPD symptoms reported, and the number of puffs/day of rescue medication during the 52 -week treatment.

\section{Sample Size and Statistical Analyses}

A total of 615 patients with a clinical diagnosis of COPD and moderate or severe airflow limitation were randomized (1:1:1) into the 3 treatment groups (Figure 1). The primary endpoint was analyzed based on the Safety set (all patients that received at least 1 dose of study drug and had at least 1 post-baseline safety assessment). The safety assessment included all safety measurements, such as AEs and COPD exacerbations. All treatment-emergent AEs starting on or after the time of the first administration of the study drug but not later than 7 days (30 days in the case of a serious adverse event [SAE]) after the last administration, were summarized. Serious cardiac or cerebrovascular (CCV) AEs and deaths were adjudicated by an independent adjudication committee. All AEs were coded according to the Medical Dictionary for Regulatory Activities (MedDRA; version 17.0). No statistical hypothesis testing was performed on AEs.

A composite endpoint of major adverse cardiovascular events (MACE) was included in the analysis, which represented all serious CCV AEs adjudicated by an independent committee to one of the following categories: a)non-fatal myocardial infarction, b)hospitalization for unstable angina, c)non-fatal stroke, d)heart failure requiring hospitalization, and e) coronary re-vascularization (coronary artery bypass graft or percutaneous coronary intervention).

Particular attention was paid to the LABA and LAMA drug classes in terms of safety variables, such as increased heart rate, increased blood pressure, CCV (major cerebrovascular events, cardiac arrhythmias),

\section{Figure 1: Trial Profile of the Flight3 Study}

\section{FLIGHT3}

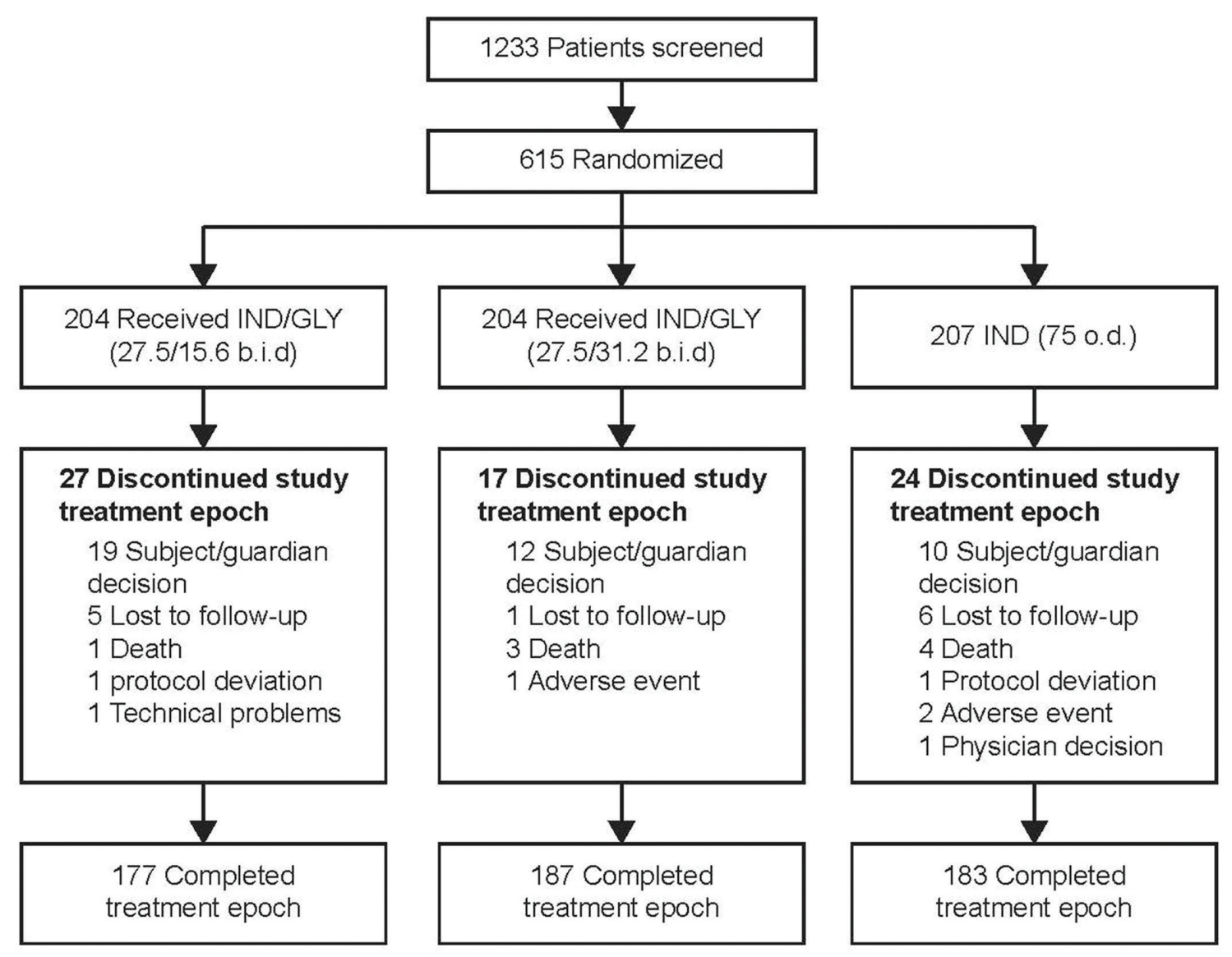

b.i.d., twice daily; GLY, glycopyrronium; IND, indacaterol; o.d., once daily 
hypokalemia, diabetes and hyperglycemia, corrected QT interval prolongation, paradoxical bronchospasm, narrow-angle glaucoma, blurred vision, urinary outflow obstruction, urinary retention, and anticholinergic syndrome.

The change from baseline in pre-dose trough $\mathrm{FEV}_{1}$ from Week 4 to Week 52 was analyzed using a mixedmodel repeated-measures (MMRM) analysis on a full analysis set (FAS; all randomized patients who received at least 1 dose of study medication). This model included treatment, baseline $\mathrm{FEV}_{1}$, visit, treatment by visit interaction, visit by baseline $\mathrm{FEV}_{1}$ interaction, smoking status at baseline, baseline ICS use, airflow limitation severity, and region as fixed effects with unstructured variance-covariance error matrix. Similar analysis was performed for change from baseline in post-dose 1-h $\mathrm{FEV}_{1}$ (as measured at the same visits and at Day 1), predose trough FVC, and post-dose 1-h FVC.

The time to first COPD exacerbation was assessed using Kaplan-Meier curve and analyzed using Cox regression model for the FAS. The rate of COPD exacerbations was analyzed using a generalized linear model assuming a negative binomial distribution. The $\log$ (exposure time) was used as an offset variable in the model.

Daily, morning, and evening symptom scores were analyzed using a linear mixed model (LMM). A similar LMM was also used to analyze daily, daytime, and nighttime rescue mediation uses. The change from baseline in COPD assessment test (CAT) total score at Weeks 12, 28, 44, and 52 was analyzed using a similar MMRM as that used for pre-dose trough $\mathrm{FEV}_{1}$, with baseline $\mathrm{FEV}_{1}$ replaced by baseline CAT total score.

\section{Results}

\section{Patients}

Of the 615 patients randomized to treat, 85.2\% completed the planned study treatment. One randomized patient in the IND group did not receive the study medication and was excluded from the analysis. Overall, 68 patients discontinued the treatment, with the most frequent reason for discontinuation being patient/guardian decision $(n=41)$ (Figure 1). Other reasons for treatment discontinuation included lack of efficacy, protocol deviations, and physician's decision (with comparable rates among treatment groups) (Figure 1). Most patients (>99\%) were compliant with the study medication per protocol (which categorized compliance as $80 \%-100 \%$ ) with a similar rate between the twice daily and once daily treatment groups.

Patient demographics were comparable between the 3 treatment groups (Table 1).The majority of the patients were male, and nearly all patients were white. (Approximately 60\% of patients were classified as Group B and approximately $40 \%$ as Group D according to GOLD 2011 strategy $^{24}$ (Table 1). Since an mMRC score of $\geq 2$ was required for study entry, no Group A or $C$ patients were included. In the year prior to enrollment, only $33 \%$ patients had a history of COPD exacerbation (Table 1).

\section{Outcomes}

\section{Adverse Events}

The incidence of AEs was similar across the treatment

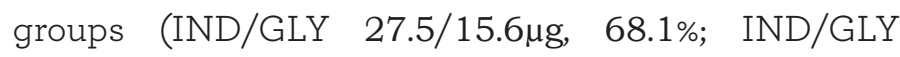

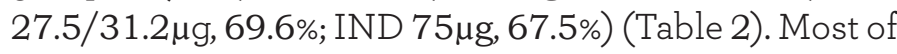
the reported AEs were mild (22.1\%-25.7\%) or moderate (30.6\%-35.3\%) in severity. COPD exacerbation was the most frequently occurring $\mathrm{AE}$, and the $\mathrm{AE}$ rate was similar across the treatment groups (IND/GLY

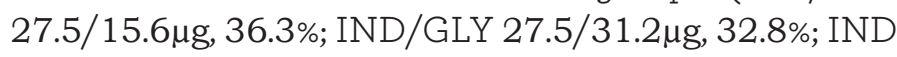
$75 \mu \mathrm{g}, 36.9 \%$ ) (Table 2 ). The rate of discontinuation from the study treatment due to AEs was lowest for IND/GLY

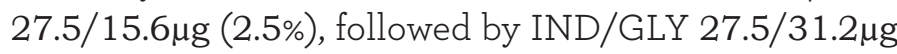
(3.9\%) and IND $75 \mu \mathrm{g}(5.8 \%)$. The 3 treatment groups demonstrated comparable trends when assessing the time to first AEs leading to permanent discontinuation (Figure 2). The incidence of drug-related AEs was higher with IND $75 \mu \mathrm{g}(9.2 \%)$ than that with IND/GLY

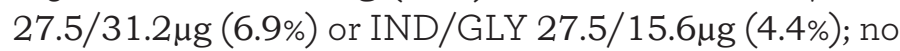
specific pattern of drug-related discontinuations was observed.

The incidence of SAEs was low and comparable between all treatment groups: IND/GLY 27.5/15.6 $\mu \mathrm{g}$,

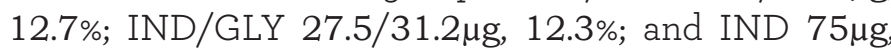
$11.7 \%$ (Table 2). Similar trends were observed between the treatment groups when assessing the time to first SAE (including COPD exacerbation) (Figure 3). The rate of discontinuation of the study medication due to SAEs was also low and comparable between all

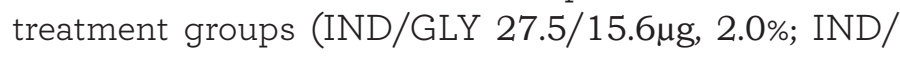

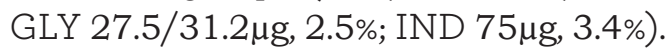

The incidence of CCV events as a risk category was

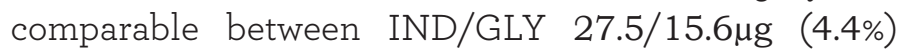
and IND $75 \mu \mathrm{g}(4.4 \%)$ but was higher with IND/GLY

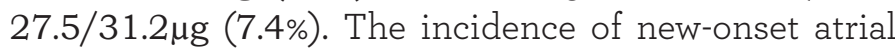
fibrillation/flutter events was comparable between IND/ 


\section{Table 1. Patient Demographics and Baseline Characteristics in the FLIGHT3 Study (Randomized Set)}

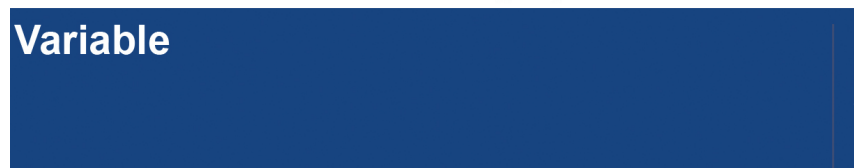

\section{IND/GLY \\ $27.5 / 15.6 \mu \mathrm{g}$ \\ b.i.d. $n=204$}

\section{IND/GLY \\ $27.5 / 31.2 \mu \mathrm{g}$ \\ b.i.d. $n=204$}

$\begin{array}{cc}\substack{\text { IND } \\ 75 \mu \mathrm{g} \text { o.d. } \\ \mathrm{n}=207} & \text { Total } \\ \mathrm{N}=615\end{array}$

Male, n (\%)

Race, n (\%) White Other

Duration of COPD, years

Severity of airflow limitation (GOLD stage), n (\%)

Moderate (GOLD II) Severe (GOLD III)

GOLD classification GOLD B

GOLD D

ICS users at baseline, $\mathrm{n}(\%)$

Smoking history, n (\%) Ex-smoker Current smoker

Number COPD exacerbations in the previous year, $n(\%)$ 1 $\geq 2$

mMRC dyspnea scale, $\mathrm{n}(\%)$ Grade 2 Grade 3 Grade 4

CAT score categorized, $\mathrm{n}(\%)$ 0-10 (mild) 11-20 (moderate) 21-30 (severe) 31-40 (very severe)

Pre-bronchodilator FEV 1 , L

Post-bronchodilator FEV $1, \mathrm{~L}$

Post-bronchodilator FEV 1 , (\% FEV 1 predicted)

FEV $_{1}$ reversibility, \%

Post-bronchodilator FEV 1 /FVC, \%

Cardiovascular risk factor

CCV history/condition

Hypertension

Hyperlipidemia

Diabetes mellitus

BMI $>30 \mathrm{~kg} / \mathrm{m}^{2}$

Age $\geq 65$ years

Current smoker at baseline

Data are presented as mean \pm standard deviation, unless stated otherwise;

COPD severity is based on GOLD 2011 criteria
$63.9(8.50)$

$123(60.3)$

$131(64.2)$

$62.8(8.52) \quad 63.8(8.32)$

$149(72.0) \quad 403(65.5)$

$199(97.5)$

5 (2.5)

6.7 (5.28)

128 (62.7)

73 (35.8)

$118(57.8)$

83 (40.7)

95 (46.6)

202 (99.0)

2 (1.0)

$6.8(5.48)$

200 (96.6)

$601(97.7)$

7 (3.4)

$6.6(5.07)$

$14(2.3)$ $6.7(5.27)$

103 (50.5)

101 (49.5)

78 (38.2)

129 (62.3)

381 (62.0) $74(35.7)$ 225 (36.6) (\%)

141 (69.1)

49 (24.0)

14 (6.9)

\section{5 (56.4)}

$120(58.0)$

353 (57.4)

87 (42.6)

83 (40.1)

253 (41.1)

99 (48.5)

$101(48.8)$

295 (48.0)

$141(69.1)$

60 (29.4)

3 (1.5)

99 (48.5)

100 (48.3)

$302(49.1)$

105 (51.5)

107 (51.7)

313 (50.9)

$141(69.1)$

$141(69.1)$
$46(22.5)$

131 (63.3)

$60(29.0)$

$413(67.2)$

$17(8.3)$

$16(7.7)$

155 (25.2)

47 (7.6)

\begin{tabular}{r|r|r}
\hline $141(69.1)$ & $127(61.4)$ & $409(66.5)$ \\
\hline $61(29.9)$ & $73(35.3)$ & $194(31.5)$ \\
\hline $2(1.0)$ & $7(3.4)$ & $12(2.0)$ \\
\hline
\end{tabular}

46 (22.5)

$102(50.0)$

50 (24.5)

6 (2.9)

$1.254(0.4772)$

1.505 (0.5030)

55.0 (13.18)

21.8 (14.78)

49.8 (10.81)

$32(15.7)$
$93(45.6)$
$73(35.8)$
$6(2.9)$

31 (15.0)

109 (17.7)

112 (54.1)

307 (49.9)

58 (28.0)

181 (29.4)

\begin{tabular}{|c|c|c|}
\hline $6(2.9)$ & $6(2.9)$ & 18 (2.9) \\
\hline $1.232(0.4540)$ & $1.278(0.4566)$ & $1.255(0.4623)$ \\
\hline $1.479(0.4849)$ & $1.551(0.4910)$ & $1.512(0.4931)$ \\
\hline $54.2(12.62)$ & $53.9(11.78)$ & $54.4(12.52)$ \\
\hline $23.2(16.10)$ & $24.0(17.98)$ & $23.0(16.35)$ \\
\hline 48.7 (10.73) & 49.4 (10.09) & $49.3(10.54)$ \\
\hline
\end{tabular}

\section{3 (11.3)}

111 (54.4)

72 (35.3)

21 (10.3)

61 (29.9)

91 (44.6)

101 (49.5)

$22(10.8)$
$118(57.8)$
$61(29.9)$
$21(10.3)$
$63(30.9)$
$100(49.0)$
$105(51.5)$

19 (9.2)

$104(50.2)$

$74(35.7)$

27 (13.0)

62 (30.0)

90 (43.5)

107 (51.7)

64 (10.4)

333 (54.1)

207 (33.7)

69 (11.2)

186 (30.2)

281 (45.7)

313 (50.9)
$124(60.8)$

b.i.d.: twice daily; BMI: body mass index; CCV: cardiac or cerebrovascular; COPD: chronic obstructive pulmonary disease;

CAT: chronic obstructive pulmonary disease assessment test; $\mathrm{FEV}_{1}$ : forced expiratory volume in 1 second; FVC: forced vital capacity;

GOLD: Global initiative for chronic Obstructive Lung Disease; GLY: glycopyrronium; IND: indacaterol, ICS: inhaled corticosteroid;

mMRC: modified Medical Research Council; o.d.: once daily 


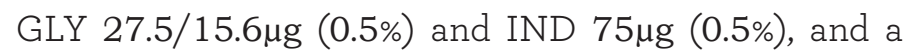
$0.0 \%$ event rate was reported for IND/GLY 27.5/31.2 ug. The incidence of any AEs of special interest (CCV and non-CCV) was comparable across the treatment groups

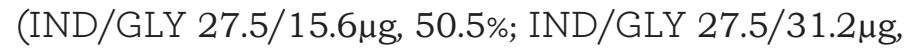
52.9\%; IND $75 \mu \mathrm{g}, 47.6 \%)$. The most frequently reported AEs of special interest were COPD exacerbation (IND/

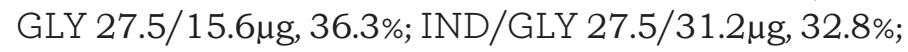
IND $75 \mu \mathrm{g}, 36.9 \%$ ) and AEs related to the respiratory composite endpoint of upper and lower respiratory tract infections, pneumonia, bronchitis (IND/GLY

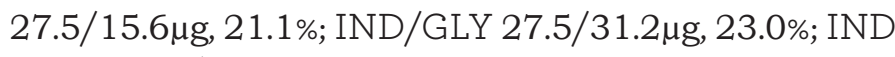
$75 \mu \mathrm{g}, 23.8 \%)$.

The number of MACE and/or CV deaths were also comparable across the treatment groups: IND/GLY

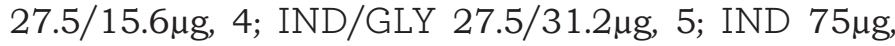
3. Overall, 9 patients died during the study: IND/GLY

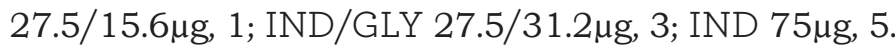
The majority of the deaths were cardiovascular-related. Importantly, only 1 death was reported over 52 weeks on

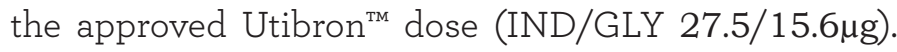
None of the deaths were considered to be related to the

\section{Table 2. Safety Analysis From FLIGHT3 Study (Safety Set)}

\begin{tabular}{|c|c|c|c|}
\hline & $\begin{array}{l}\text { IND/GLY } \\
27.5 / 15.6 \mu g \\
\text { b.i.d. n=204 }\end{array}$ & 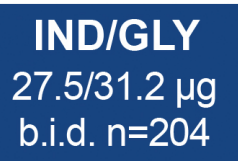 & $\begin{array}{c}\text { IND } \\
75 \mu g \text { o.d. } \\
n=206\end{array}$ \\
\hline Patients with at least $1 \mathrm{AE}, \mathrm{n}(\%)$ & $139(68.1)$ & $142(69.6)$ & $139(67.5)$ \\
\hline \multicolumn{4}{|l|}{ Any $A E$ events in $\geq 1.0 \%$ of Any Group } \\
\hline COPD Exacerbation & $74(36.3)$ & $67(32.8)$ & $76(36.9)$ \\
\hline Nasopharyngitis & $19(9.3)$ & $18(8.8)$ & $22(10.7)$ \\
\hline Upper Respiratory Tract Infection Bacterial & $12(5.9)$ & $14(6.9)$ & $13(6.3)$ \\
\hline Back Pain & $10(4.9)$ & $7(3.4)$ & $5(2.4)$ \\
\hline Upper Respiratory Tract Infection & $10(4.9)$ & $10(4.9)$ & $9(4.4)$ \\
\hline Lower Respiratory Tract Infection & $9(4.4)$ & $5(2.5)$ & $6(2.9)$ \\
\hline Pneumonia & $7(3.4)$ & $4(2.0)$ & $2(1.0)$ \\
\hline Viral Upper Respiratory Tract Infection & $7(3.4)$ & $6(2.9)$ & $7(3.4)$ \\
\hline Diarrhea & $5(2.5)$ & $2(1.0)$ & $3(1.5)$ \\
\hline Headache & $5(2.5)$ & $7(3.4)$ & $4(1.9)$ \\
\hline Hypertension & $5(2.5)$ & $10(4.9)$ & $4(1.9)$ \\
\hline Sinusitis & $5(2.5)$ & $8(3.9)$ & $6(2.9)$ \\
\hline Death & $1(0.5)$ & $3(1.5)$ & $5(2.4)$ \\
\hline $\mathbf{S A E}(\mathbf{s})$ & $26(12.7)$ & $25(12.3)$ & $24(11.7)$ \\
\hline Cardiac Disorders & $4(2.0)$ & $8(3.9)$ & $4(1.9)$ \\
\hline Respiratory and Mediastinal Disorders & $11(5.4)$ & $5(2.5)$ & $12(5.8)$ \\
\hline Patients with at Least 1 Adjudicated Serious CCV AE & $7(3.4)$ & $9(4.4)$ & $3(1.5)$ \\
\hline MACE* & $3(1.5)$ & $4(2.0)$ & 0 \\
\hline Non-MACE Serious CCV AE* & $4(2.0)$ & $5(2.5)$ & $3(1.5)$ \\
\hline New-onset Adjudicated Atrial Fibrillation/flutter Events & $1(0.5)$ & 0 & $1(0.5)$ \\
\hline Discontinuation of Treatment Due to $\mathrm{AE}(\mathrm{s})$ & $5(2.5)$ & $8(3.9)$ & $12(5.8)$ \\
\hline Discontinuation of Treatment Due to SAE(s) & $4(2.0)$ & $5(2.5)$ & $7(3.4)$ \\
\hline Discontinuation of Treatment Due to Non-SAE(s) & $1(0.5)$ & $4(2.0)$ & $7(3.4)$ \\
\hline AEs Requiring Dose Interruption & $8(3.9)$ & $5(2.5)$ & $8(3.9)$ \\
\hline AEs Requiring Additional Therapy & $108(52.9)$ & $114(55.9)$ & $111(53.9)$ \\
\hline
\end{tabular}

*Adjudicated

Data are presented as $n(\%)$

AE: adverse event; COPD: chronic obstructive pulmonary disease; SAE: serious adverse event; MACE: major adverse cardiovascular events; CCV: cardiac or cerebrovascular 


\section{Figure 2. Time to First AE (Including COPD Exacerbations Leading to Permanent Discontinuation of Study Drug)}

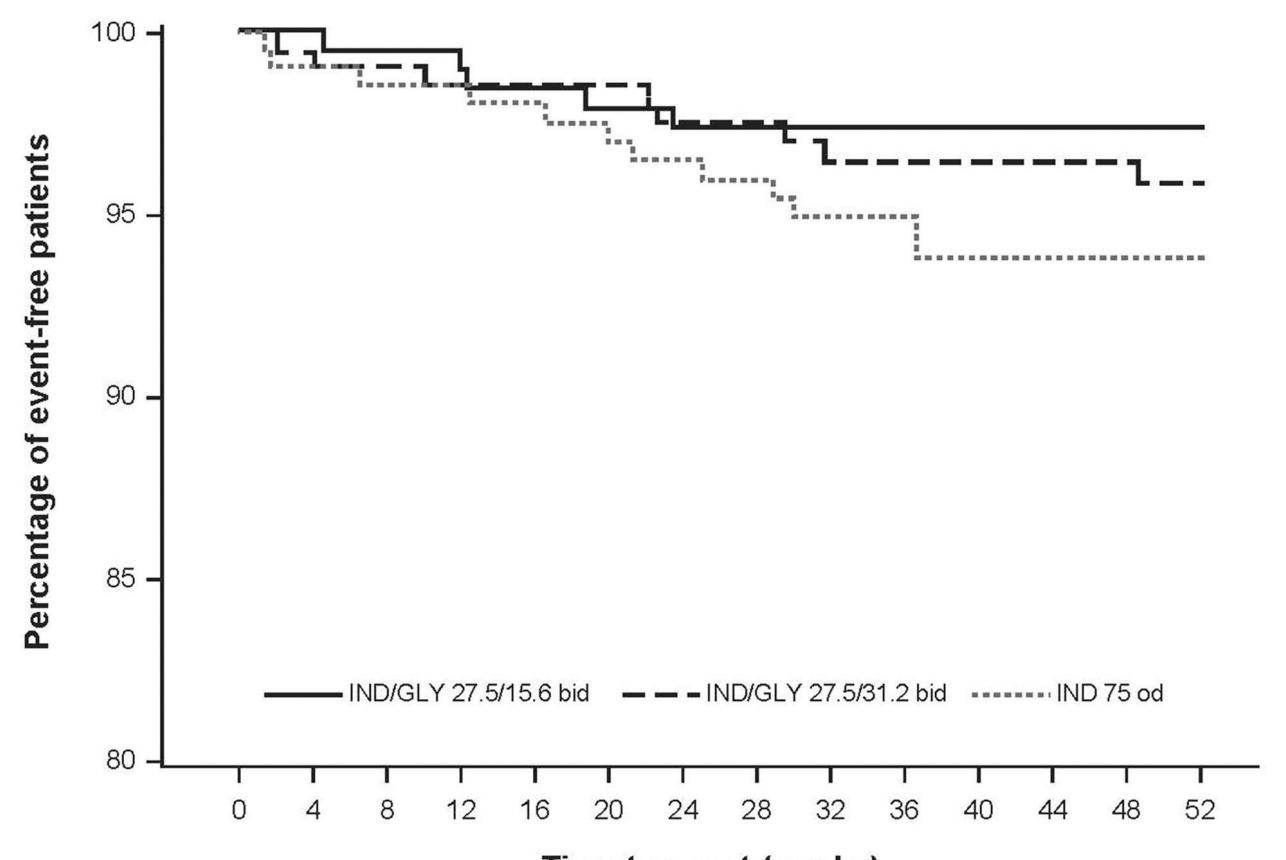

Numbers at risk/failed

$\begin{array}{lllll}\text { IND/GLY 27.5/15.6 } & 204 & 190 / 2 & 177 / 5 & 130 / 5 \\ \text { IND/GLY 27.5/31.2 } & 204 & 194 / 3 & 189 / 5 & 127 / 8 \\ \text { IND 75 } & 206 & 196 / 3 & 182 / 8 & 130 / 12\end{array}$

Patients who did not discontinue study drug prematurely for an AE were censored at the date they completed study treatment. Figure truncated after week 52 (Day 365)

$\mathrm{AE}$, adverse event; b.i.d., twice daily; COPD, chronic obstructive pulmonary disease; GLY, glycopyrronium; IND, indacaterol; o.d., once daily

study drug by the reporting investigator.

No clinically meaningful between-group differences for any clinical chemistry or hematological parameter were observed. ECG parameters did not demonstrate any meaningful between-group differences in the rates of abnormalities seen for Fridericia-corrected QT interval values and other ECG intervals. There was no evidence of a treatment-related effect on vital signs (pulse and systolic and diastolic blood pressures) (see online supplemental data eTables 2 and 3 ).

\section{Trough FEV 1 and FVC}

Improvements in pre-dose trough $\mathrm{FEV}_{1}$ with both the IND/GLY doses were consistently superior to those with IND at Week 52 (treatment difference $[\Delta], 80$

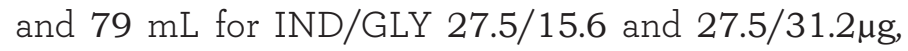
respectively) at all visits up to Week 52 (Figure 4[a]). Improvements in post-dose 1 -h $\mathrm{FEV}_{1}$ with IND/GLY $27.5 / 15.6 \mu \mathrm{g}$ were consistently superior to those with
IND $75 \mu \mathrm{g}$ at all visits up to 52 weeks $(\Delta, 44 \mathrm{~mL}$ on Day 1; $\Delta, 108 \mathrm{~mL}$ on Day 365) (Figure 4 [b]). Similarly, predose trough FVC and post-dose 1-h FVC with both the IND/GLY doses were superior to those with IND over 52 weeks (online supplemental data eFigures 1 and 2).

\section{COPD Exacerbation and Patient-reported Endpoints}

The number of patients who experienced mild, moderate, or severe COPD exacerbations was comparable among the treatment groups. Both IND/GLY doses resulted in a $12 \%$ reduction in the risk of moderate or severe COPD exacerbation compared with IND, although this was not statistically significant (hazard ratio [HR], 0.88 and $p=0.516$ for IND/GLY 27.5/15.6 $\mu$ g versus IND $75 \mu \mathrm{g}$; HR, 0.88 and $p=0.502$ for IND/GLY 27.5/31.2 $\mu \mathrm{g}$ versus IND $75 \mu \mathrm{g})$. There were numerical betweengroup differences in the rate of moderate or severe COPD exacerbations, with IND/GLY 27.5/15.6 $\mu \mathrm{g}$ demonstrating $25 \%$ fewer exacerbations versus IND 


\section{Figure 3. Time to First SAE (Including COPD Exacerbations)}

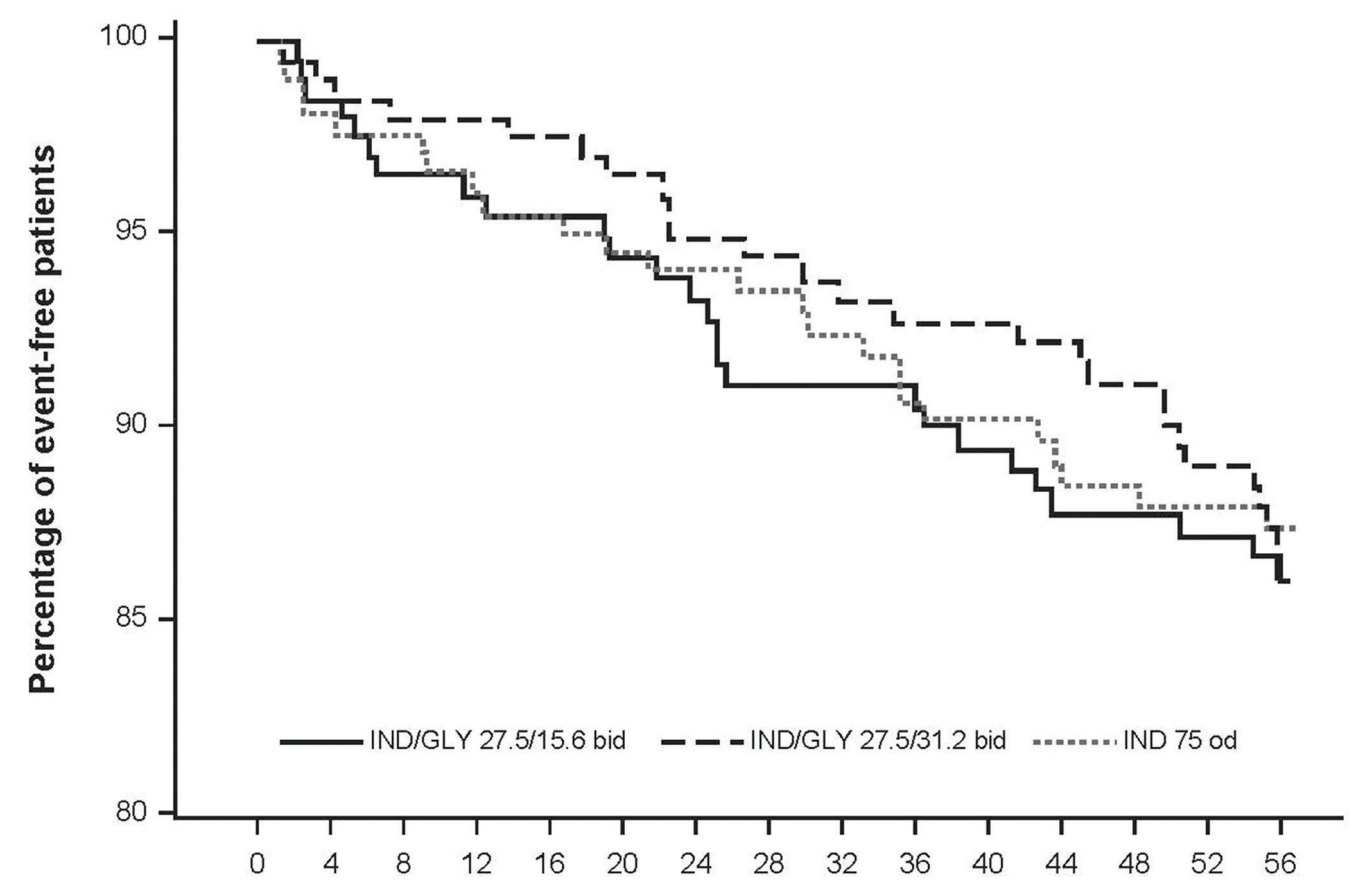

Time to event (weeks)

\section{Numbers at risk/failed}

$\begin{array}{lll}\text { IND/GLY 27.5/15.6 } & 204 & 184 / 8 \\ \text { IND/GLY 27.5/31.2 } & 204 & 192 / 4 \\ \text { IND 75 } & 206 & 191 / 8\end{array}$

$167 / 17$
$182 / 11$
$173 / 13$

$173 / 13$

$162 / 20$
$173 / 14$
$163 / 19$

$158 / 24$

$165 / 21$

$159 / 23$

Patients who did not experience an event were censored at the earlier date of last treatment day+ 30 days and final visit. Figure truncated at Week 56 (Day 365)

b.i.d., twice daily; COPD, chronic obstructive pulmonary disease; GLY, glycopyrronium; IND, indacaterol; o.d., once daily; SAE, serious adverse event

$75 \mu$ g (rates ratio, 0.75 and $p=0.163$ for IND/GLY $27.5 / 15.6 \mu \mathrm{g}$ versus IND $75 \mu \mathrm{g}$; rates ratio, 0.87 and $p=0.464$ for IND/GLY 27.5/31.2 $\mu$ g versus IND $75 \mu \mathrm{g})$. A majority of symptom score endpoints, use of rescue medication, and CAT scores were similar between the treatment groups (online supplemental data eTables 4 and 5 and eFigure 3 ).

\section{Discussion}

In this 1-year study, the safety and efficacy of IND/ GLY, a b.i.d. LABA/LAMA combination therapy, was compared with that of IND, a LABA, in COPD patients with moderate-to-severe airflow limitation. We believe that this is the first time that a 52 -week LABA/LAMA (b.i.d) safety study is being reported in the United States. Overall, $14.8 \%$ of patients discontinued the study treatment. The range of discontinuation rates was lower than that observed in other 52-week COPD studies $(30 \%-40 \%){ }^{25-26}$ The rate of discontinuation of the study treatments due to $\mathrm{AEs}$ was lowest for IND/GLY 27.5/15.6 $\mu$ g b.i.d., followed by IND/GLY $27.5 / 31.2 \mu \mathrm{g}$ b.i.d. and IND $75 \mu \mathrm{g}$ o.d. Fewer patients on IND/GLY 27.5/15.6 $\mu$ b.i.d. discontinued treatment compared with those on IND $75 \mu$ g o.d.; however, this difference was not clinically significant. The incidence of MACE and/or CV deaths was also comparable across the treatment groups. The incidence of CCV events as a risk category was comparable between IND/GLY $27.5 / 15.6 \mu \mathrm{g}$ b.i.d. and IND $75 \mu \mathrm{g}$ o.d. and was higher with IND/GLY 27.5/31.2 $\mu$ g b.i.d. The overall incidence of AEs and SAEs was similar between the treatment groups. There were no clinically meaningful differences in laboratory tests, ECG, and vital signs. A majority of symptom score endpoints, use of rescue medication, and CAT scores were similar between the treatment groups. The AEs, SAEs, and the overall safety profile 


\section{Figure 4. (a) Pre-dose Trough FEV 1 (L), Change from Baseline (FAS)}

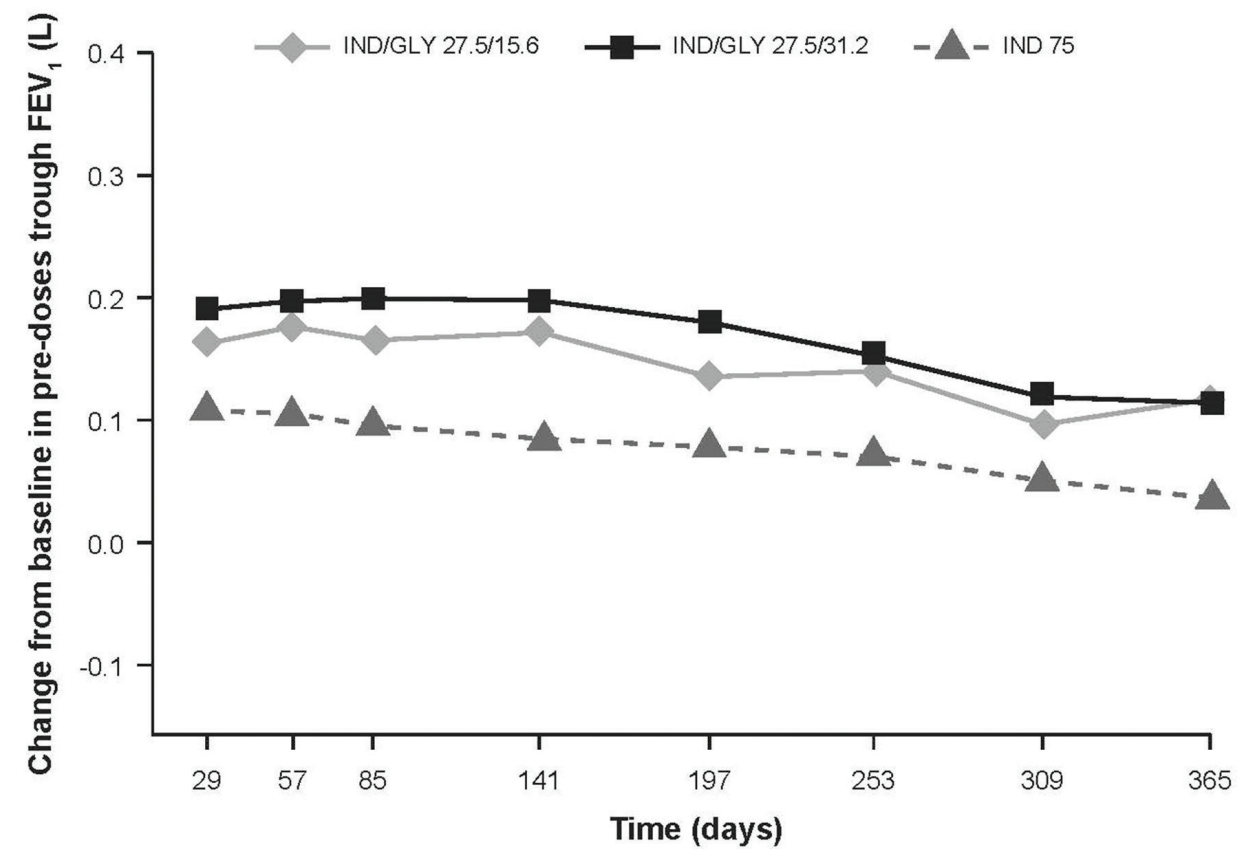

MMRM: Change from baseline in pre-dose trough $\mathrm{FEV}_{1}=$ treatment + baseline $\mathrm{FEV}_{1}+$ smoking status at baseline + baseline

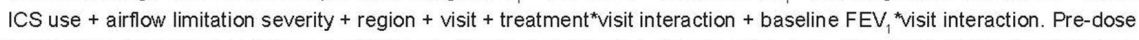
trough $\mathrm{FEV}$ is defined as the mean of $\mathrm{FEV}_{1}$ at $-45 \mathrm{~min}$ and $-15 \mathrm{~min}$ before morning dose. Baseline is defined as the average of the pre-dose $\mathrm{FEV}_{1}$ measured at $-45 \mathrm{~min}$ and $-15 \mathrm{~min}$ at Day 1 .

b.i.d., twice daily; FAS, full analysis set; GLY, glycopyrronium; IND, indacaterol; ICS, inhaled corticosteroid; MMRM, mixed-model repeated-measures; $F E V_{1}$, forced expiratory volume in $1 \mathrm{~s}$; o.d., once daily

\section{(b) FEV 1 (1 hour post-dose), Change from Baseline (FAS)}

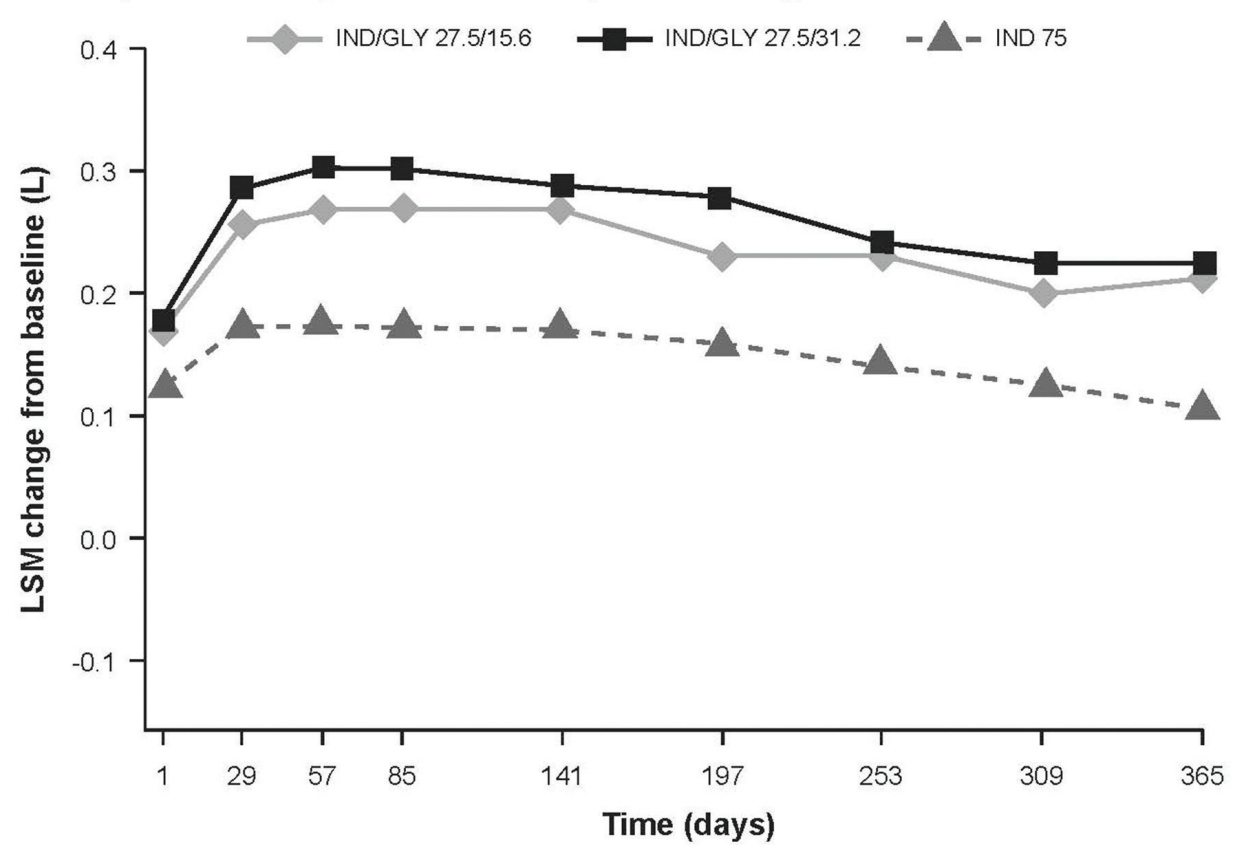

MMRM: Change from baseline in pre-dose trough $\mathrm{FEV}_{1}=$ treatment + baseline $\mathrm{FEV}_{1}+$ smoking status at baseline + baseline ICS use + airflow limitation severity + region + visit + treatment ${ }^{*}$ visit interaction + baseline FEV ${ }_{1}^{*}$ visit interaction

b.i.d., twice daily; FAS, full analysis set; $F_{1} V_{1}$, forced expiratory volume in $1 \mathrm{~s}$; GLY, glycopyrronium; LSM, least squares mean; IND, indacaterol; ICS, inhaled corticosteroid; MMRM, mixed-model repeated-measures; o.d., once daily 
of IND/GLY over 1 year were similar to those in other IND/GLY studies of 12 -week durations. ${ }^{27}$

The safety profiles of FDCs of LABA/LAMAs are of key importance, particularly because earlier analyses examining the CV safety profile of these drug classes have raised questions related to their safety. ${ }^{28,29}$ This study contained 2 different doses of GLY to alleviate safety concerns typically associated with LAMA

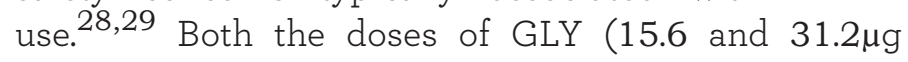
b.i.d.) were safe and well tolerated over 52 weeks, with no significant differences between the doses. A pooled safety analysis of a higher IND/GLY dose

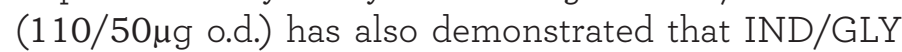
has a safety profile comparable to that of a placebo. ${ }^{30}$ Importantly, a shorter-term cardiovascular safety study also demonstrated that IND/GLY had a safety profile similar to that of placebo in terms of CV assessments 31 and that IND/GLY was well tolerated, with overall AE rates similar to those with a placebo. ${ }^{31}$ A pooled analysis of the 6-month safety data extrapolated from 4 trials (SHINE, ILLUMINATE, ENLIGHTEN, and ARISE) on the o.d. 110/50 ug dosage also demonstrated that the proportion of COPD patients treated with IND/GLY who experienced CCV events was similar to the proportion of such patients treated with tiotropium and lower than the proportion of such patients receiving placebo. ${ }^{32}$ Recently, the FLIGHT1 and FLIGHT2 studies have demonstrated that the safety and tolerability profile of IND/GLY in patients receiving IND/GLY 27.5/15.6 $\mu \mathrm{g}$ is comparable to those of the monocomponents IND and GLY or placebo after 12 weeks. ${ }^{27}$ Compared with the short-term FLIGHT1 and FLIGHT2 studies, the FLIGHT3 study demonstrated the long-term safety of IND/GLY versus IND over a longer treatment period (52-week).

The effect of IND/GLY on pulmonary function was evaluated as a secondary objective. In the pooled FLIGHT studies, statistically superior bronchodilation was observed with IND/GLY than that with its monocomponents or placebo. ${ }^{27}$ In this study, the 1-year efficacy data on pulmonary function again demonstrated that the patients receiving IND/GLY had a superior long-term bronchodilation compared with patients receiving IND. Pre-dose trough FEV 1 and post-dose 1-h $\mathrm{FEV}_{1}$ were consistently superior with IND/GLY 27.5/15.6 $\mu$ g versus IND $75 \mu \mathrm{g}$ at all visits over 52 weeks, indicating maintenance of lung function and persistence of efficacy. Both the doses of IND/GLY achieved approximately $80 \mathrm{~mL}$ in trough $\mathrm{FEV}_{1}$ versus
IND alone. In addition, the onset of efficacy was quick as $\mathrm{FEV}_{1}$ (post-dose 1 -h) was $44 \mathrm{~mL}$ on Day 1 and $108 \mathrm{~mL}$ on Day 365 for IND/GLY versus IND alone.

Although this study was neither designed nor powered as an exacerbation trial with high-risk patients, there were numerical between-group differences in the rate of reduction of moderate or severe COPD exacerbations, with IND/GLY 27.5/15.6ug demonstrating 25\% fewer exacerbations as compared with IND $75 \mu \mathrm{g}$. In addition, both the IND/GLY doses resulted in a $12 \%$ reduction in the risk of moderate or severe COPD exacerbations as compared with IND. Although these exacerbation results were not statistically significant, they are suggestive of a possible added therapeutic benefit if an appropriately designed and powered study is to be performed.

There are limitations of this study that have to be acknowledged. This study had no placebo group due to the long-term nature of the study. It was considered unethical to recruit patients with moderate-to-severe COPD in a placebo group for a 52 -week duration. No significant difference was observed between IND/GLY $27.5 / 15.6 \mu \mathrm{g}$ and IND $75 \mu \mathrm{g}$ in terms of exacerbations/ symptoms possibly because both the treatments were highly active, and the study was not powered to demonstrate this.

In conclusion, the FLIGHT3 study demonstrated the long-term safety, tolerability, and sustained efficacy of IND/GLY 27.5/15.6 $\mu$ g b.i.d. in patients with moderateto-severe COPD over 52 weeks of treatment. This data demonstrates that IND/GLY b.i.d. is a safe and welltolerated treatment option for long-term use in patients with COPD.

\section{Acknowledgements}

The authors thank Sinead Flannery and David Bergin for their assistance in the preparation of the manuscript. Authors' contributions: GF, AFT, AS-T, FP and DB participated in study design and concept of the study. Statistical analyses were overseen by $\mathrm{CT}$ and QW. All the authors contributed to the acquisition and interpretation of data and also in drafting and revision of the manuscript.

\section{Declaration of Interest}

GF is supported in part by multiple pharmaceutical clinical research projects, including grants from Novartis Pharmaceuticals, Corp. AFT, AST, FP and DB are employees of Novartis Pharmaceuticals Corp. 


\section{References}

1. Global initiative for chronic Obstructive Lung Disease (GOLD). Global strategy for the diagnosis, management, and prevention of chronic obstructive pulmonary disease. GOLD website. http:// www.goldcopd.org/uploads/users/files/GOLD_Report_2015_. pdf Published 2015 Accessed May 3, 2015.

2. Blasi F, Cesana G, Conti S, et al. The clinical and economic impact of exacerbations of chronic obstructive pulmonary disease: a cohort of hospitalized patients. PloS One. 2014;9(6):e101228. doi: http://dx.doi.org/10.1371/journal.pone.0101228

3. Miravitlles M, Sicras A, Crespo C, et al. Costs of chronic obstructive pulmonary disease in relation to compliance with guidelines: a study in the primary care setting. Ther Adv Respir Dis. 2013;7(3):139-150.

doi: http://dx.doi.org/10.1177/1753465813484080

4. Rutten-van Molken MP and Goossens LM. Cost effectiveness of pharmacological maintenance treatment for chronic obstructive pulmonary disease: a review of the evidence and methodological issues. Pharmacoeconomics. 2012;30(4):271-302.

doi: http://dx.doi.org/10.2165/11589270-000000000-00000

5. National Institutes of Health. National Heart Lung and Blood Institute (NHLBI). NHLBI Chartbook 2012. NHLBI website. http://www.nhlbi.nih.gov/files/docs/research/2012_ChartBook _508.pdf Published February 2012. Accessed April 21, 2015

6. Murphy SL, Kochanek KL, Xu J, Heron M. Deaths: final data for 2012. Natl Vital Stat Rep. 2015; 63(9):1-117.

7. Centers for Disease Control and Prevention. Chronic obstructive pulmonary disease among adults-United States, 2011. MMWR Morb Mortal Wkly Rep. 2012;61(46):938-943.

8. American Lung Association. State of Lung Disease in Diverse Communities 2010. American Lung Association website. http://action.lung.org/site/DocServer/state-of-lung-diseasein-diverse-communities-2010.pdf?docID $=87442010$ Published 2010. Accessed May 2016.

9. van Noord JA, Aumann JL, Janssens E, et al. Effects of tiotropium with and without formoterol on airflow obstruction and resting hyperinflation in patients with COPD. Chest. 2006;129(3):509517. doi: http://dx.doi.org/10.1378/chest.129.3.509

10. Vogelmeier C, Kardos P, Harari S, Gans SJ, Stenglein S, Thirlwell J. Formoterol mono- and combination therapy with tiotropium in patients with COPD: a 6-month study. Respir Med. 2008;102(11):1511-1520.

doi: http://dx.doi.org/10.1016/j.rmed.2008.07.020

11. Cazzola M and Molimard M. The scientific rationale for combining long-acting $\beta 2$-agonists and muscarinic antagonists in COPD. Pulm Pharmacol Ther. 2010;23(4):257-267. doi: http://dx.doi.org/10.1016/j.pupt.2010.03.003
12. Cazzola M and Tashkin DP. Combination of formoterol and tiotropium in the treatment of COPD: effects on lung function. COPD. 2009;6(5):404-415.

doi: http://dx.doi.org/10.1080/15412550903156333

13. Tashkin DP, Donohue JF, Mahler DA, et al. Effects of arformoterol twice daily, tiotropium once daily, and their combination in patients with COPD. Respir Med. 2009;103(4):516-524. doi: http://dx.doi.org/10.1016/j.rmed.2008.12.014

14. van Noord JA, Aumann JL, Janssens E, et al. Comparison of tiotropium once daily, formoterol twice daily and both combined once daily in patients with COPD. Eur Respir J. 2005;26(2):214222. doi: http://dx.doi.org/10.1183/09031936.05.00140404

15. van Noord JA, Aumann JL, Janssens E, et al. Combining tiotropium and salmeterol in COPD: Effects on airflow obstruction and symptoms. Respir Med. 2010;104(7):995-1004. doi: http://dx.doi.org/10.1016/j.rmed.2010.02.017

16. Chapman KR, Rennard SI, Dogra A, Owen R, Lassen C, Kramer B. Long-term safety and efficacy of indacaterol, a long-acting beta(2)-agonist, in subjects with COPD: a randomized, placebocontrolled study. Chest. 2011;140(1):68-75. doi: http://dx.doi.org/10.1378/chest.10-1830

17. D'Urzo A, Ferguson GT, van Noord JA, et al. Efficacy and safety of once-daily NVA237 in patients with moderate-to-severe COPD: the GLOW1 trial. Respir Res. 2011;12:156. doi: http://dx.doi.org/10.1186/1465-9921-12-156

18. Beeh KM, Singh D, Di SL, Drollmann A. Once-daily NVA237 improves exercise tolerance from the first dose in patients with COPD: the GLOW3 trial. Int J Chron Obstruct Pulmon Dis. 2012;7:503-513. doi: http://dx.doi.org/10.2147/COPD.S32451

19. Kerwin E, Hebert J, Gallagher N, et al. Efficacy and safety of NVA237 versus placebo and tiotropium in patients with COPD: the GLOW2 study. Eur Respir J. 2012;40(5):1106-1114. doi: http://dx.doi.org/10.1183/09031936.00040712

20. Yu AP, Guerin A, de Leon DP, et al. Clinical and economic outcomes of multiple versus single long-acting inhalers in COPD. Respir Med. 2011;105(12):1861-1871. doi: http://dx.doi.org/10.1016/j.rmed.2011.07.001

21. Bateman ED, Ferguson GT, Barnes N, et al. Dual bronchodilation with QVA149 versus single bronchodilator therapy: the SHINE study. Eur Respir J. 2013;42(6):1484-1494.

doi: http://dx.doi.org/10.1183/09031936.00200212

22. Verkindre C, Fukuchi Y, Flemale A, et al. Sustained 24-h efficacy of NVA237, a once-daily long acting muscarinic antagonist, in COPD patients. Respir Med. 2010;104(10):1482-1489. doi: http://dx.doi.org/10.1016/j.rmed.2010.04.006

23. Arievich H, Overend T, Renard D, et al. A novel model-based approach for dose determination of glycopyrronium bromide in COPD. BMC Pulm Med. 2012;12:74. doi: http://dx.doi.org/10.1186/1471-2466-12-74 
24. Global Initiative for Chronic Obstructive Lung Disease (GOLD). Global strategy for the diagnosis, management, and prevention of chronic obstructive pulmonary disease, revised 2011. GOLD website. http://www.goldcopd.org/uploads/users/files/ GOLD2011_Summary.pdf Published November 2011. Accessed May 2016.

25. D’Urzo A, Kerwin E, Rennard S, He T, Gil EG, Caracta C. Oneyear extension study of ACCORD COPD I: safety and efficacy of two doses of twice-daily aclidinium bromide in patients with COPD. COPD. 2013;10(4):500-510.

doi: http://dx.doi.org/10.3109/15412555.2013.791809

26. Donohue JF, Niewoehner D, Brooks J, O’Dell D, Church A. Safety and tolerability of once-daily umeclidinium/vilanterol 125/25 mcg and umeclidinium $125 \mathrm{mcg}$ in patients with chronic obstructive pulmonary disease: results from a 52-week, randomized, double-blind, placebo-controlled study. Respir Res. 2014; $15: 78$.

27. Mahler DA, Kerwin E, Ayer T, et al. Efficacy and safety of QVA 149 compared with its mono-components and placebo in moderateto-severe COPD patients: The FLIGHT studies. The FLIGHT studies. Am J Respir Crit Care Med. 2015;192(9):1068-1079. doi: http://dx.doi.org/10.1164/rccm.201505-1048OC

28. Rossi A, Khirani S, Cazzola M. Long-acting beta2-agonists (LABA) in chronic obstructive pulmonary disease: efficacy and safety. Int J Chron Obstruct Pulm Dis. 2008;3(4):521-529.

29. Singh S, Loke YK, Furberg CD. Inhaled anticholinergics and risk of major adverse cardiovascular events in patients with chronic obstructive pulmonary disease: a systematic review and metaanalysis. JAMA. 2008;300(12):1439-1450.

doi: http://dx.doi.org/10.1001/jama.300.12.1439

30. Wedzicha JA, Dahl R, Buhl R, et al. Pooled safety analysis of the fixed-dose combination of indacaterol and glycopyrronium (QVA 149), its mono-components, and tiotropium versus placebo in COPD patients. Respir Med. 2014;108(10):1498-1507. doi: http://dx.doi.org/10.1016/j.rmed.2014.07.011

31. Van de Maele B, Fabbri LM, Martin C, Horton R, Dolker M, Overend T. Cardiovascular safety of QVA149, a combination of Indacaterol and NVA237, in COPD patients. COPD. 2010;7(6):418-427.

doi: http://dx.doi.org/10.3109/15412555.2010.528812

32. Ferguson G, Barnes N, Mehta R. Cardio- and cerebro-vascular safety profile of QVA 149 in patients with COPD: a pooled analysis [Abstract]. Am J Respir Crit Care Med. 2013;187(1):A1488. 